\title{
Anterior selective stabilization combined with laminoplasty for cervical myelopathy due to massive ossification of the posterior longitudinal ligament: report of early outcomes in 14 patients
}

\author{
Yukitaka Nagamoto, MD, PhD, Motoki Iwasaki, MD, PhD, Shinya Okuda, MD, PhD, \\ Tomiya Matsumoto, MD, PhD, Tsuyoshi Sugiura, MD, PhD, Yoshifumi Takahashi, MD, and \\ Masayuki Furuya, MD, PhD
}

Department of Orthopaedic Surgery, Osaka Rosai Hospital, Sakai, Japan

OBJECTIVE Surgical management of massive ossification of the posterior longitudinal ligament (OPLL) is challenging. To reduce surgical complications, the authors have performed anterior selective stabilization combined with laminoplasty (antSS+LP) for massive OPLL since 2012. This study aimed to elucidate the short-term outcome of the antSS+LP procedure.

METHODS The authors' analysis was based on data from 14 patients who underwent antSS+LP for cervical myelopathy caused by massive OPLL and were followed up for at least 2 years after surgery (mean follow-up duration 3.3 years). Clinical outcome was evaluated preoperatively, at 6 months and 1 year postoperatively, and at the final follow-up using the Japanese Orthopaedic Association (JOA) scoring system for cervical myelopathy and the recovery rate of the JOA score. The following radiographic parameters were measured preoperatively, immediately after surgery, at 1 year after surgery, and at the final follow-up: the C2-7 angle, measured on lateral plain radiographs, and the segmental lordosis angle (SLA), measured on sagittal CT scans. The correlation between radiographic parameters and clinical outcomes was evaluated.

RESULTS The mean JOA score increased from 10.4 before surgery to 13.6 and 13.8 at 6 months and 1 year after surgery, respectively; at the final follow-up the mean score was 13.4 . This postoperative recovery was significant $(p=0.004)$ and was maintained until the final follow-up. No patient required revision surgery due to postoperative neurological deterioration. However, the C2-7 angle gradually deteriorated postoperatively. Similarly, the SLA was significantly increased immediately after surgery, but the improvement was not maintained. The recovery rate at the final follow-up correlated positively with the change in C2-7 angle $(r=0.60, p=0.03)$ and the change in $S L A(r=0.72, p<0.01)$.

CONCLUSIONS AntSS+LP is safe and effective and may be an alternative to anterior decompression and fusion for the treatment of patients with massive OPLL. No postoperative neurological complications or significant postoperative exacerbation of neck pain were observed in our case series. Not only reducing intervertebral motion and decompressing the canal at the maximal compression level but also acquiring segmental lordosis at the maximal compression level are crucial factors for achieving successful outcomes of antSS+LP.

https://thejns.org/doi/abs/10.3171/2020.1.SPINE191068

KEYWORDS ossification of posterior longitudinal ligament; cervical myelopathy; laminoplasty; posterior decompression with fusion; anterior decompression with fusion

$\mathrm{O}$ SSIFICATION of the posterior longitudinal ligament (OPLL) is an ectopic ossification that can cause spinal cord compression and result in myelopathy. Surgical spinal decompression is widely accepted as a treatment option to resolve the myelopathy. ${ }^{18}$ Although favorable surgical outcomes following laminoplasty can be expected in most cases of OPLL, the surgical management for massive OPLL, with an occupying ratio $>60 \%$ and hill-shaped ossification, is challenging. ${ }^{37,10,21}$ In cases of massive OPLL, anterior decompression and fusion

ABBREVIATIONS ADF = anterior decompression and fusion; antSS = anterior selective stabilization; EBL = estimated blood loss; JOA = Japanese Orthopaedic Association; LP = laminoplasty; NRS = numeric rating scale; OPLL = ossification of the posterior longitudinal ligament; PDF = posterior decompression and fusion; SLA = segmental lordosis angle.

SUBMITTED September 5, 2019. ACCEPTED January 7, 2020.

INCLUDE WHEN CITING Published online March 13, 2020; DOI: 10.3171/2020.1.SPINE191068. 
(ADF) can ideally resolve spinal cord compression by direct removal of the ossification and can provide superior surgical outcome to any other procedure. However, ADF has tended to fall into disfavor because of its technically demanding nature and its high incidence of perioperative complications. Posterior decompression and fusion (PDF) using instrumentation can provide favorable outcomes, even for massive OPLL and, therefore, is becoming popular as a safe alternative to ADF. However, the complications of PDF, such as upper-limb palsy and neck stiffness and pain, cannot be overlooked.

In our practice, we have performed anterior selective stabilization combined with laminoplasty (antSS+LP) for the treatment of massive OPLL since 2012, except in patients with an extensive continuous type of mass having a low dynamic factor and/or rigid kyphosis. The antSS+LP procedure aims to immobilize dynamic factors at the level of maximal compression via an anterior approach and to decompress the static compression factor via a posterior approach. The procedure can be expected to be useful for reducing the risk of upper-limb palsy and neck stiffness by restoring cervical range of motion. The purpose of this study was to elucidate the short-term outcomes of the antSS+LP procedure.

\section{Methods}

The study was a retrospective case series analysis, approved by the research ethics committee of Osaka Rosai Hospital.

From 2012 to 2018, 16 consecutive patients underwent antSS+LP for cervical myelopathy due to massive OPLL. All patients were followed up after surgery. Of the 16 eligible patients, 14 had completed their 2-year follow-up at the time of data analysis. Due to the timing of their surgery, 2 other patients had not yet reached the 2-year endpoint of follow-up at the time of analysis and, therefore, their data were not included. The study group included 11 men and 3 women, with a mean duration of follow-up of 3.3 years (range $2-7$ years). The mean age at the time of surgery was 59.6 years (range 46-77 years). The mean occupying ratio of the OPLL was $60.7 \%$ (range 50\%-76\%), with 11 cases presenting with a mixed-type OPLL, 2 with a localized OPLL, and 1 with a segmental OPLL.

\section{Surgical Indication and Procedures}

The antSS+LP technique was indicated for patients with cervical myelopathy due to OPLL who satisfied the following criteria: 1) $\geq 50 \%$ canal occupying ratio; 2) obvious focal spinal cord compression as represented by hill-type ossification; and 3) minute gap between the interrupted ossifications at the level of maximal compression. Regarding the second item, a hill-shaped ossification was identified in 13 patients and a plateau-shaped ossification in the remaining patient. The one plateau-type ossification case had a slightly longer focal compression area and required double-level stabilization. Regarding the third item, the existence of a minute gap on sagittal CT scanning is always indicative of remaining segmental motion at the intervertebral level. ${ }^{3}$ AntSS+LP was not indicated for patients with no minute gap at the level of
1. Anterior interbody fusion

2. Laminoplasty

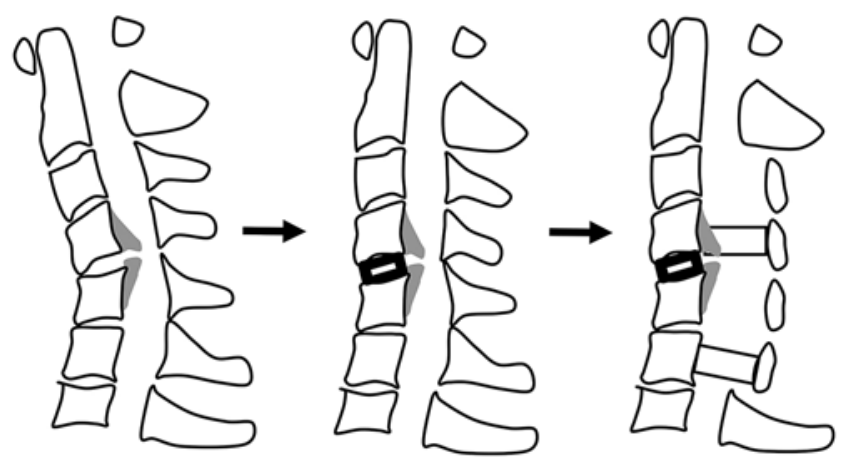

FIG. 1. Procedural steps for antSS+LP. First, anterior interbody fusion was performed at the maximal compression level without resecting the ossification, with open-door laminoplasty performed in the second step.

maximal compression and/or in those with a rigid cervical kyphosis.

For all procedures (Fig. 1), antSS was performed first. The concept of antSS is to obtain neurological improvement by removing the dynamic factor at the maximal compression level. To avoid complications that can arise from resection of the ossification, antSS does not aim to decompress the neural elements anteriorly. Patients with remaining segmental motion at the level of maximal compression were appropriate for antSS+LP, as previously noted, and the maximal compression level was always chosen for antSS. The standard left-sided Smith-Robinson method for antSS was used in all cases. Sufficient discectomies were performed, including excision of the annulus and cartilaginous endplate to achieve an effective interbody arthrodesis, while the posterior longitudinal ligament and/ or ossified ligament were left without excision. Intervertebral cages (PEEK Prevail [Medtronic Sofamor Danek] in the first 2 cases and SynCage C [DePuy Synthes]), filled with autogenous cancellous bone from the anterior iliac crest, were then implanted by applying distraction force between the vertebrae, leaving the posterior longitudinal ligament and/or ossified ligament as they were. Cages with a height slightly higher than the preoperative disc height were chosen to maintain the intervertebral distraction and achieve segmental lordosis. Of note, antSS is not intended to correct cervical kyphosis. The distribution of fused levels across patients in our study group was as follows: C3-4 (5 patients), C4-5 (2 patients), C5-6 (4 patients), C3-5 (2 patients), and C4-6 (1 patient). The involvement of the static compression factor could not be neglected in the development of myelopathy in patients with massive OPLL. ${ }^{13}$ Therefore, in these patients, posterior decompression was performed simultaneously. An open-door laminoplasty was performed through a standard posterior straight-incision approach, using 2 spacers as struts to prevent lamina closure. Postoperatively, the cervical spine was immobilized using a neck collar for 1 month.

\section{Outcome Measures}

Clinical outcome was evaluated preoperatively, at 6 months and 1 year postoperatively, and at the final follow- 


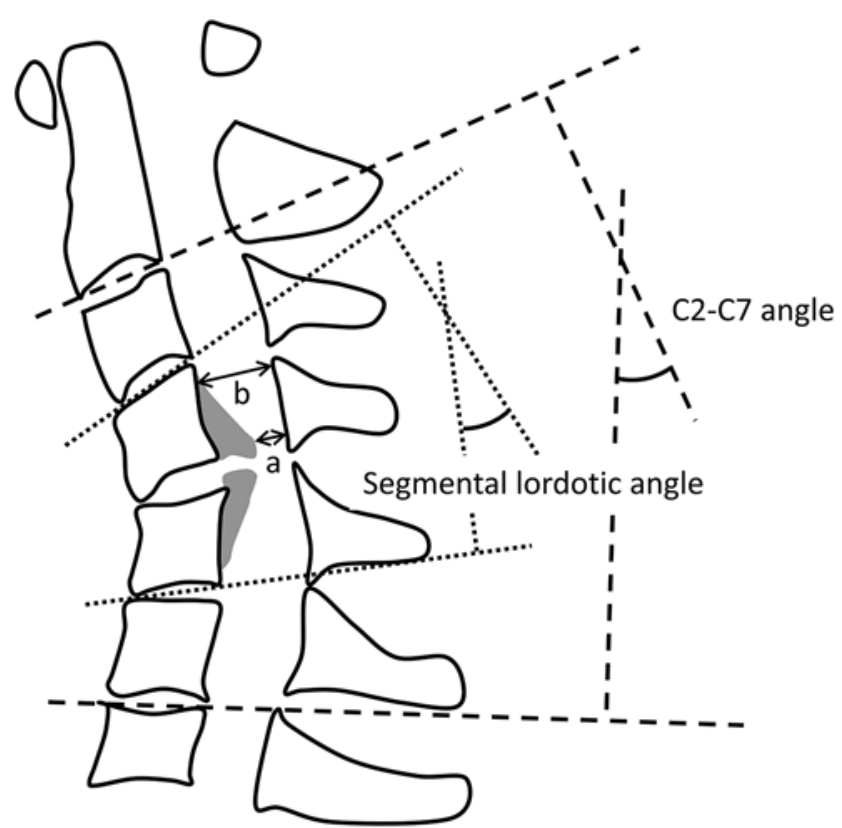

FIG. 2. Radiographic parameters. Cervical lordosis was measured between the lower endplates of $\mathrm{C} 2$ and $\mathrm{C} 7$ on lateral plain radiographs. When the $\mathrm{C} 7$ vertebra was not visible, the lower endplate of $\mathrm{C} 6$ was substituted. The SLA was measured on sagittal CT images. $a=$ space available for the spinal cord; $b=$ canal diameter.

up using the Japanese Orthopaedic Association (JOA) scoring system for cervical myelopathy ${ }^{17}$ and the recovery rate of the JOA score, calculated using the Hirabayashi method, as follows: recovery rate $(\%)=$ (postoperative $\mathrm{JOA}$ - preoperative JOA $) /(17$ - preoperative JOA $) \times 100 .^{5}$

Operative time, estimated blood loss (EBL), and perioperative complications were also obtained from the chart. The numeric rating scale (NRS) for neck pain was assessed preoperatively and at the final follow-up.

Radiographic parameters were measured preoperatively, immediately after surgery, at 1 year postoperatively, and at the final follow-up, using the C2-7 angle on lateral plain radiographs and the segmental lordosis angle (SLA) on sagittal CT images (Fig. 2). The amount of change from preoperative to final follow-up values was also measured ( $\triangle \mathrm{C} 2-7$ and $\triangle \mathrm{SLA}$, respectively). Progression of the ossification, defined as an increase of more than $2 \mathrm{~mm}$, and bridging of the minute gap were evaluated on CT at the final follow-up. The correlation between radiographic parameters and clinical outcomes was evaluated.

\section{Statistical Analysis}

To assess the statistical difference in JOA score and radiographic parameters between periods, Friedman's test was used. This was followed by a Dunn-Bonferroni test for pairwise comparisons when the overall Friedman test was statistically significant at the 0.05 level. The significance of the change in NRS score for neck pain from the preoperative assessment to final follow-up was assessed using the Wilcoxon signed-rank test. Pearson's correlation was used to assess the statistical correlation between radiographic parameters and clinical outcomes. For all
TABLE 1. Mean JOA scores and recovery rates

\begin{tabular}{lcccc}
\hline & Preop & 6 Mos & 1 Yr & Final FU \\
\hline JOA score & 10.4 & 13.6 & 13.8 & 13.4 \\
\hline RR $(\%)$ & & 47.7 & 49.6 & 45.0 \\
\hline
\end{tabular}

$\mathrm{FU}=$ follow-up; $\mathrm{RR}=$ recovery rate.

The postoperative recovery from baseline was statistically significant at 6 months $(p=0.004), 1$ year $(p=0.002)$, and final follow-up $(p<0.001)$. The Wilcoxon signed-rank test was used to compare the difference of the recovery rate at each interval.

tests, $\mathrm{p}<0.05$ was considered significant. All statistical analyses were conducted using IBM SPSS Statistics for Windows (version 21, IBM Corp.).

\section{Results}

The mean operative time was 247 minutes with a mean EBL of $179 \mathrm{ml}$. Regarding perioperative complications, 1 patient required reintubation due to pharyngeal edema. The patient was extubated 5 days after the surgery, without any further complication. There was no incidence of postoperative upper-limb palsy.

The mean JOA score was 10.4 (range 6-14.5) preoperatively, and it improved to 13.6 (range 9.5-16) at 6 months, remaining relatively stable thereafter, at 13.8 (range 11.516) at 1 year postoperatively, and 13.4 (range 9-16) at the final follow-up. The mean JOA recovery rate was $47.7 \%$ (range $0 \%-81 \%$ ) at 6 months, $49.6 \%$ (range 17\%-81\%) at 1 year postoperatively, and $45.0 \%$ (range 6\%-69\%) at the final follow-up (Table 1). The postoperative recovery was statistically significant $(\mathrm{p}=0.004)$ and was maintained through to the final follow-up. No patient required revision surgery due to postoperative neurological deterioration. The mean NRS score for neck pain was 3.1 (range 0-4) preoperatively and 2.0 (range 0-5) at the final follow-up, with this change not being significant $(\mathrm{p}=0.41)$.

The mean C2-7 angle was $5.1^{\circ}$ (range $-8^{\circ}$ to $29^{\circ}$ ) preoperatively, $3.1^{\circ}$ (range $-23^{\circ}$ to $33^{\circ}$ ) immediately after surgery, $2.2^{\circ}$ (range $-29^{\circ}$ to $24^{\circ}$ ) at 1 year postoperatively, and $2.0^{\circ}$ (range $-26^{\circ}$ to $29^{\circ}$ ) at the final follow-up (Fig. 3). Although the $\mathrm{C} 2-7$ angle tended to deteriorate after surgery, differences across the time points of measurement were not significant. The mean SLA was $0.3^{\circ}$ (range $-5^{\circ}$ to $6^{\circ}$ ) preoperatively, $8.7^{\circ}$ (range $-2^{\circ}$ to $18^{\circ}$ ) immediately after surgery, $3.1^{\circ}$ (range $-17^{\circ}$ to $17^{\circ}$ ) at 1 year, and $2.1^{\circ}$ (range $-17^{\circ}$ to $13^{\circ}$ ) at the final follow-up. Bone union was confirmed in all cases at 1 year postoperatively. Although the SLA was significantly increased immediately after surgery, the value could not be maintained after surgery. Among 16 fusion segments, in 14 cases, progression of the ossification was observed in a cranial direction in 3 cases, and bridging of the minute gaps was observed in 9 segments.

Regarding the correlation between radiographic parameters and clinical outcomes, the recovery rate at the final follow-up positively correlated with $\Delta \mathrm{C} 2-7$ angle $(\mathrm{r}$ $=0.60, \mathrm{p}=0.03$ ) and with a specific strong positive correlation with $\triangle \mathrm{SLA}(\mathrm{r}=0.72, \mathrm{p}<0.01$; Fig. $4 \mathrm{~A}$ and $\mathrm{B})$. The $\triangle \mathrm{SLA}$ tended to positively correlate with $\triangle \mathrm{C} 2-7$ angle ( $\mathrm{r}$ 


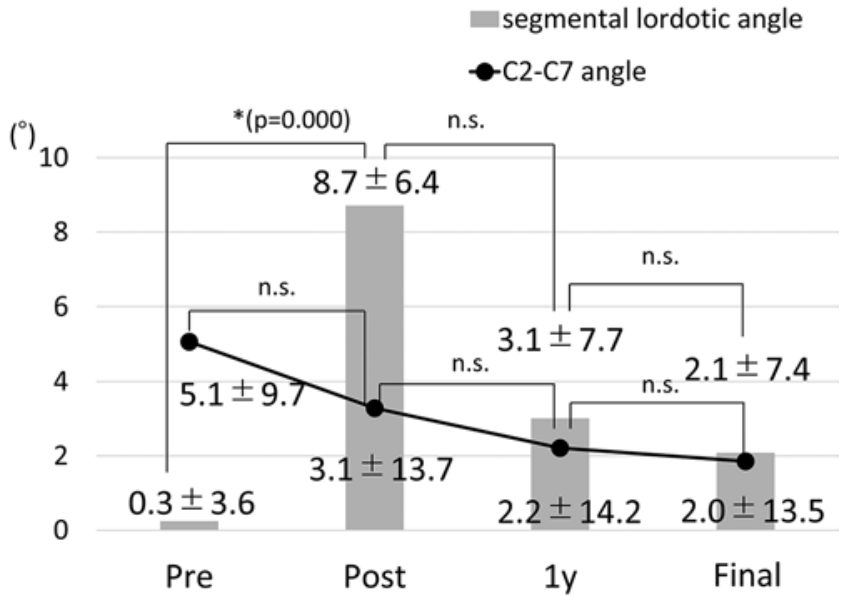

FIG. 3. Bar graph showing the SLA, with the line graph showing the C2-7 angle, preoperatively, immediately after surgery, 1 year postoperatively, and at the final follow-up. ${ }^{*} p<0.05$. n.s. = not statistically significant.

$=0.52, \mathrm{p}=0.06)$. There was no significant correlation between the recovery rate and the occupying ratio $(r=-0.14$, $\mathrm{p}=0.63$ ).

\section{Illustrative Cases \\ Case 13}

A 47-year-old man with mixed-type OPLL underwent C3-4 anterior interbody fusion combined with a C3-7 laminoplasty and a C2 dome-like laminectomy (Fig. 5). OPLL occupied $60 \%$ of the canal, with hill-shaped ossification at the C3-4 level. The operative time was 261 minutes, with an EBL of $10 \mathrm{~g}$. The patient's JOA score was 9 preoperatively, 15.5 at 6 months, 15.5 at 1 year postoperatively, and 14.5 at the final follow-up. The recovery rate at the final follow-up was $68.8 \%$. The $\mathrm{C} 2-7$ angle was $-2^{\circ}$ preoperatively, $0^{\circ}$ immediately after surgery, $1^{\circ}$ at 1 year postoperatively, and $-4^{\circ}$ at the final follow-up. The SLA was $-5^{\circ}$ preoperatively, $4^{\circ}$ immediately after surgery, and $2^{\circ}$ at the final follow-up.

\section{Case 8}

A 74-year-old woman with segmental-type OPLL un- derwent C5-6 anterior interbody fusion combined with C3-6 laminoplasty (Fig. 6). OPLL occupied 53\% of the canal, with hill-shaped ossification at the C5-6 level. The operative time was 227 minutes, and the EBL was $50 \mathrm{~g}$. The patient's JOA score was 9 preoperatively, 14 at 6 months, 12 at 1 year postoperatively, and 12 at the final follow-up. The recovery rate at the final follow-up was $37.5 \%$. The C2-7 angle was $3^{\circ}$ preoperatively, $-23^{\circ}$ immediately after surgery, $-29^{\circ}$ at 1 year postoperatively, and $-26^{\circ}$ at the final follow-up. The SLA was $-2^{\circ}$ preoperatively, $-2^{\circ}$ immediately after surgery, and $-3^{\circ}$ at the final follow-up.

\section{Discussion}

In the development of myelopathy associated with cervical OPLL, both static compression factors and dynamic factors play an important role. Onari et al. pioneered the surgical concept of reducing the dynamic factor via anterior fusion. ${ }^{15}$ They performed anterior fusion without resection of the ossification mass as the first choice for cervical OPLL and reported favorable long-term results. Their study was the first to elucidate the effectiveness of reducing the dynamic factor without resecting the ossification mass in cases of OPLL with dynamic factors. Recently, Goel et al. reported favorable short-term results of stabilization without decompression by posterior instrumentation in these cases. ${ }^{4}$ However, there has been no report on surgical outcomes of stabilization alone, for massive OPLL.

Recently, PDF has been popularized as an alternative procedure to ADF for massive OPLL because of its safety and favorable surgical outcomes. ${ }^{11,23}$ Although PDF can be an effective alternative option to ADF, the technique has several disadvantages. Some previous studies have shown a higher incidence of upper-limb palsy after PDF than after ADF, ranging between $11.9 \%$ and $50 \% .^{1,14,20}$ Takemitsu et al. reported that the risk of developing an upper-limb palsy was 11.6-fold greater after PDF than laminoplasty alone. ${ }^{19}$ Takenaka et al. reported that onset of upper-limb palsy after PDF was more frequent within 2 days after surgery than at $\geq 3$ days, with a pooled prevalence of $9.0 \%$ and $4.3 \%$, respectively. ${ }^{20}$ They speculated a lag correction effect to be the cause of early-onset upper-limb palsy after $\mathrm{PDF}$, caused by iatrogenic foraminal stenosis or tension on the nerve roots. They also reported that the incidence of

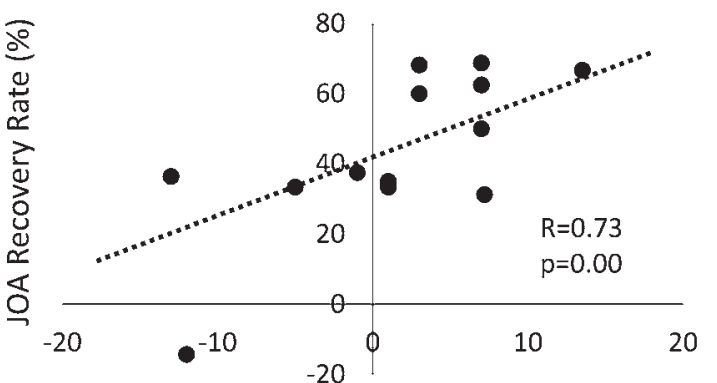

A $\triangle$ segmental lordotic angle $\left(^{\circ}\right)$

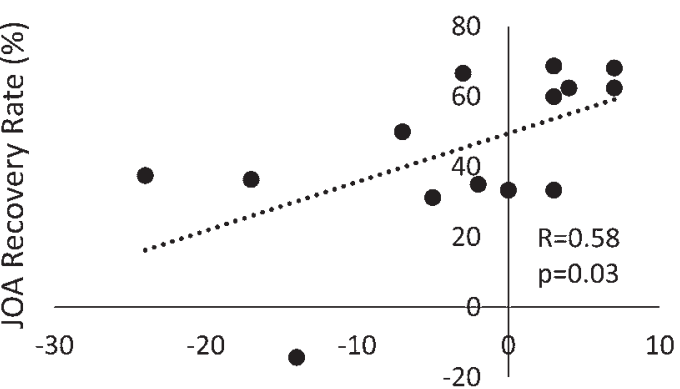

$\mathrm{B} \quad \triangle \mathrm{C} 2-\mathrm{C} 7$ angle $\left({ }^{\circ}\right)$

FIG. 4. A: Correlation between the JOA recovery rate and $\triangle$ SLA. B: Correlation between the JOA recovery rate and the change in the $\triangle \mathrm{C} 2-7$ angle. 


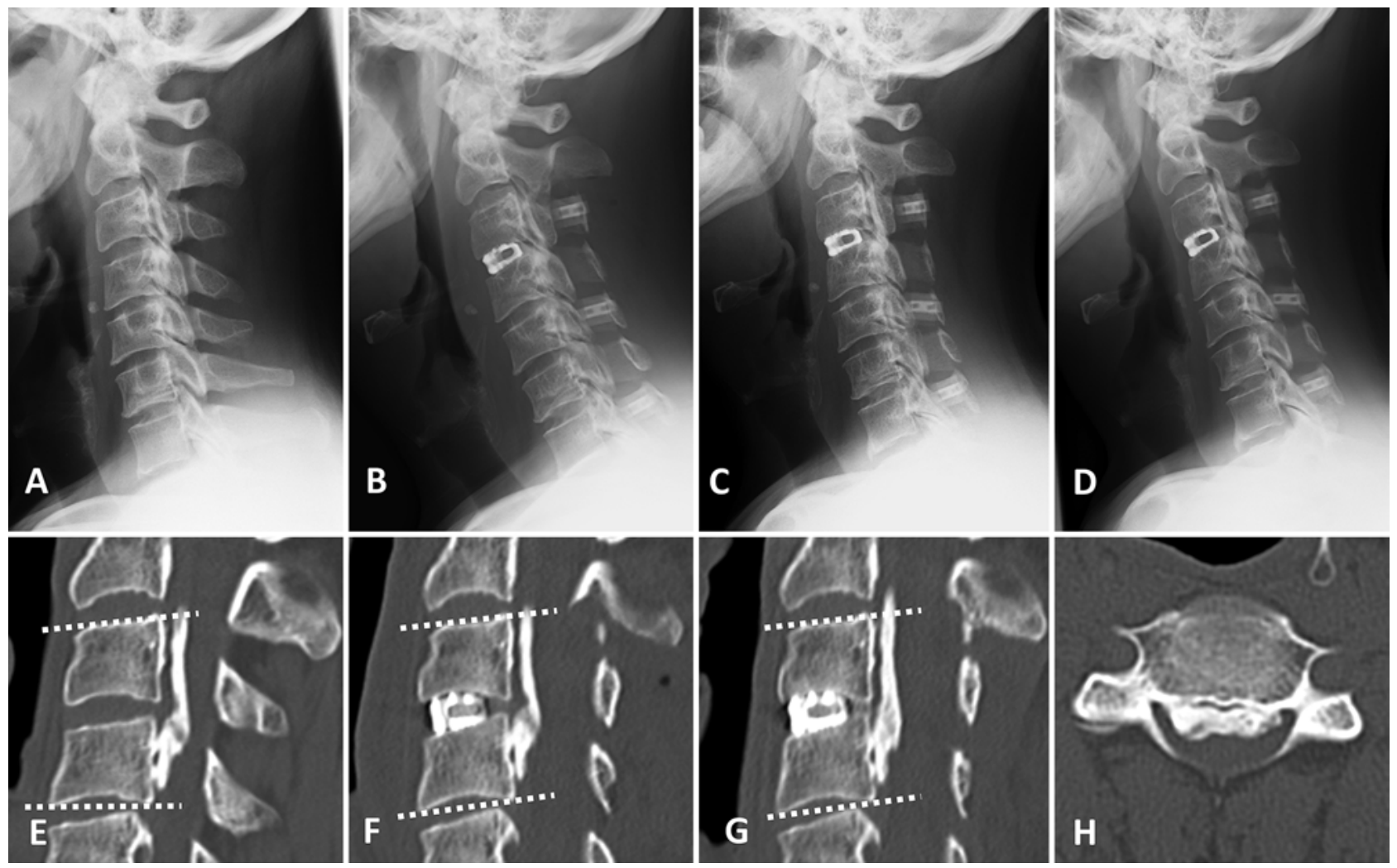

FIG. 5. Lateral radiographs obtained preoperatively (A), immediately after surgery (B), 1 year postoperatively (C), and at the final follow-up (D). Sagittal reconstructed CT images obtained preoperatively (E), immediately after surgery (F), and at the final followup (G). Axial CT scan showing the maximal compression level $(\mathbf{H})$.

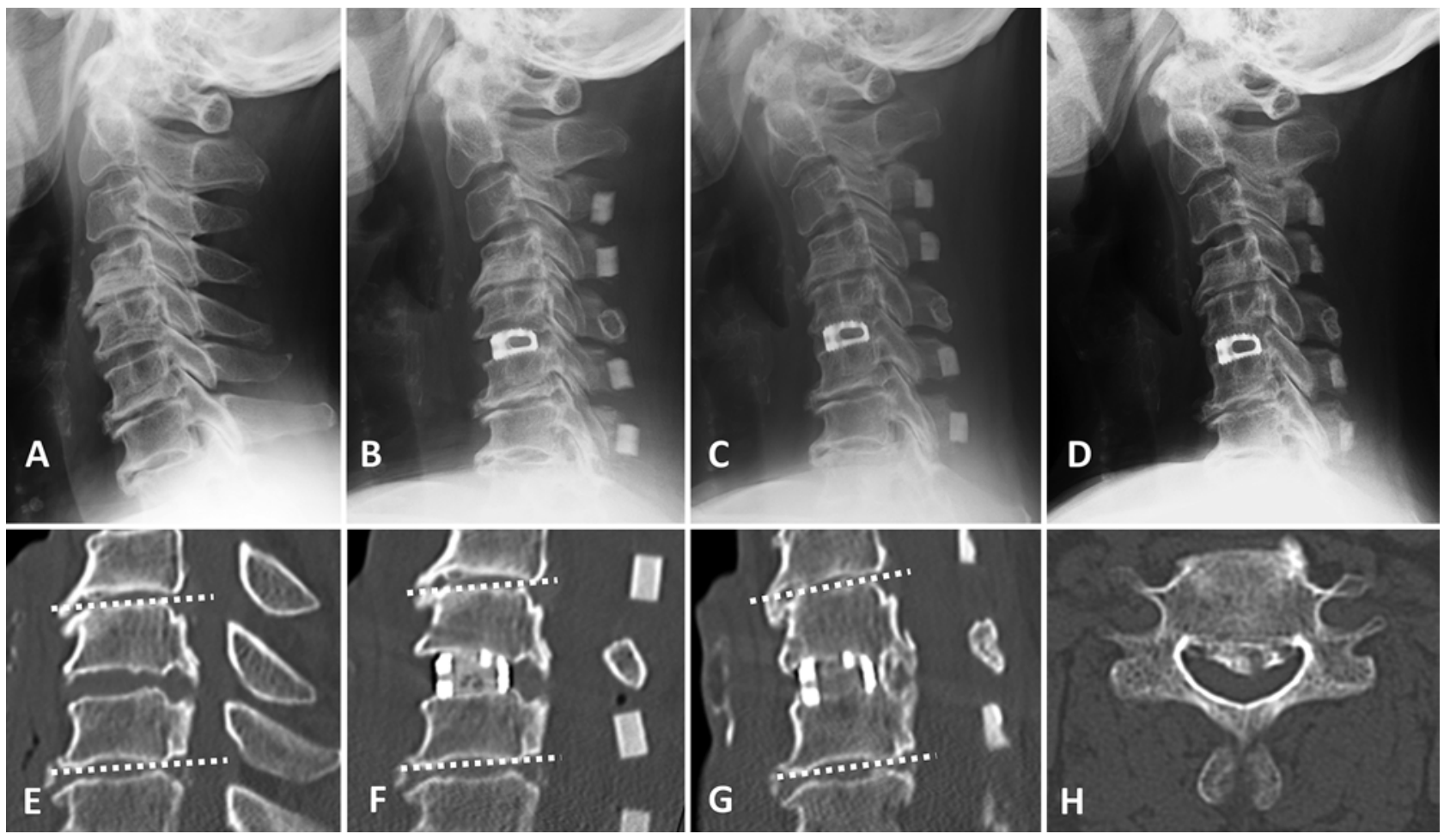

FIG. 6. Lateral radiographs obtained preoperatively (A), immediately after surgery (B), 1 year postoperatively (C), and at the final follow-up (D). Sagittal reconstructed CT images obtained preoperatively $(\mathbf{E})$, immediately after surgery $(\mathbf{F})$, and at the final followup (G). Axial CT scan showing the maximal compression level (H). 
TABLE 2. Comparison of surgical outcomes for massive OPLL among procedures

\begin{tabular}{|c|c|c|c|c|c|c|c|c|c|c|c|c|}
\hline \multirow[b]{2}{*}{ Authors \& Year } & \multirow{2}{*}{$\begin{array}{l}\text { Inclusion } \\
\text { Criterion }\end{array}$} & \multirow[b]{2}{*}{ Procedure } & \multirow{2}{*}{$\begin{array}{c}\text { No. of } \\
\text { Patients }\end{array}$} & \multirow{2}{*}{$\begin{array}{l}\text { FU } \\
\text { (yrs) }\end{array}$} & \multicolumn{3}{|c|}{ JOA Score } & \multicolumn{2}{|c|}{$\mathrm{C} 2-7 \mathrm{~A}\left({ }^{\circ}\right)$} & \multirow{2}{*}{$\begin{array}{l}\text { Op Time } \\
\text { (mins) }\end{array}$} & \multirow{2}{*}{$\begin{array}{c}\text { EBL } \\
\text { (g) }\end{array}$} & \multirow{2}{*}{$\begin{array}{l}\text { Neurological } \\
\text { Complication }\end{array}$} \\
\hline & & & & & Preop & Postop & $\mathrm{RR}(\%)$ & Preop & Postop & & & \\
\hline Iwasaki et al., 2007 & OR $>60 \%$ & LP & 6 & 10.2 & 9.4 & 11.0 & 14 & NA & NA & NA & NA & NA \\
\hline Koda et al., 2016 & K-line (-) & LP & 16 & 3.8 & 9.5 & 10.3 & 14.4 & 5.1 & -0.4 & 152 & 251 & $1(6 \%)$ \\
\hline Chen et al., 2009 & $\mathrm{OR}>50 \%$ & PDF & 28 & 4 & 8.7 & 12.4 & 43.5 & 6.5 & 11.7 & NA & NA & $4(14 \%)$ \\
\hline Saito et al., 2016 & K-line (-) & PDF & 27 & 1 & 8.0 & 11.9 & 43.6 & 2.3 & 3.1 & NA & NA & NA \\
\hline Yoshii et al., 2016 & $\mathrm{OR}>50 \%$ & PDF & 22 & 3.1 & 10.4 & 14.2 & 55.8 & 9.6 & 9.8 & 281 & 372 & $2(9 \%)$ \\
\hline Koda et al., 2016 & K-line (-) & PDF & 17 & 3.5 & 7.4 & 11.4 & 43.6 & -2.1 & -0.8 & 351 & 783 & $2(12 \%)$ \\
\hline Present study & $\mathrm{OR}>50 \%$ & AntSS+LP & 14 & 3.3 & 10.5 & 13.4 & 45.0 & 5.1 & 2.0 & 248 & 189 & $0(0 \%)$ \\
\hline
\end{tabular}

$\mathrm{NA}=$ not available; $\mathrm{OR}=$ occupying ratio.

upper-limb palsy was higher in patients with OPLL than in those without OPLL. In our case series, no incidence of postoperative upper-limb palsy was noted. Another disadvantage of PDF is neck pain after surgery. ${ }^{12,22,23}$ Postoperative neck pain after PDF has been reported to be greater than that after $\mathrm{ADF}^{23}$ and laminoplasty. ${ }^{12,22}$ Wide detachment of the paraspinal muscles is necessary to place the posterior instrumentation, with resultant injury to the paraspinal muscles being a cause of postoperative neck pain after the posterior procedure. In our case series, none of our patients reported significant postoperative exacerbation of neck pain.

Regarding surgical outcomes, neurological recovery after antSS+LP for massive OPLL may be superior to laminoplasty alone, ${ }^{7,11}$ and comparable to PDF, as shown in Table 2., ${ }^{1,11,16,23}$ The surgical invasiveness of the antSS+LP procedure, including the EBL and operative time, is also comparable to that of PDF. These favorable outcomes reflect that antSS+LP consists of a combination of simple procedures. Anterior stabilization alone is a simple procedure that does not require technically demanding procedures such as resection of the ossified mass. Laminoplasty, which is the most prevalent treatment for OPLL, is also a simple and familiar procedure for spinal surgeons. Moreover, given the absence of any upper-limb palsy in our 14 patients treated using antSS+LP, we consider the incidence of postoperative neurological complications with antSS+LP to be at least similar to either procedure alone and to be lower than that for PDF. Considering results from previous reports and the outcomes of our study, the main advantage of the antSS+LP procedure is restoration of the segmental lordosis through distraction by the implantation of an intervertebral cage. Such distraction is difficult to apply with the PDF procedure. This difference between the $\mathrm{PDF}$ and antSS+LP procedures likely explains the lower prevalence of postoperative nerve palsy, regardless of surgical outcomes. Postoperative axial progression of ossification, which has been reported in as many as 50\%-70\% of patients undergoing laminoplasty,,$^{2,6,8,9}$ was not observed in our case series. This may reflect the positive effect of anterior stabilization. However, with regard to a postoperative loss of cervical lordosis, the antSS+LP is inferior to PDF. Although the loss of cervical lordosis was small on short-term follow-up, long-term follow-up would be essential, as progression of cervical kyphosis would result in neurological deterioration. Regarding the correlation between the radiographic parameters and surgical results, the recovery rate at the final follow-up significantly correlated with the cervical sagittal alignment, with a specifically strong positive correlation with the $\triangle \mathrm{SLA}(\mathrm{r}=0.72$, $\mathrm{p}<0.01$ ). Of note, the recovery rate of the JOA score did not deteriorate significantly with the postoperative loss of the SLA. This indicates that the combination of immobilization of the dynamic factors at the level of maximal compression and decompression of the static compression factor might be the main contributor to postoperative clinical improvement, with the $\triangle$ SLA likely to be a secondary contributor.

The limitations of our study include the small number of patients in the analysis and the relatively short follow-up period of observation. In addition, due to the small number of patients we encountered, inclusion of a control arm was not feasible. Regardless of these limitations, we believe that this report is very important because the cases of massive OPLL are scarce. To confirm the effectiveness of the technique, a multicenter comparative study with other possible surgical options, such as PDF, would be warranted, using a larger number of cases with a longer follow-up.

\section{Conclusions}

AntSS+LP may provide a safe and effective alternative to ADF for the treatment of patients with massive OPLL. No postoperative neurological complications or significant postoperative exacerbation of neck pain was observed in our small case series. Not only reducing intervertebral motion and decompressing the canal at the maximal compression level but also acquiring segmental lordosis at the maximal compression level are crucial factors for successful outcomes with this technique.

\section{Acknowledgments}

This work was supported by a grant-in-aid from the Investigation Committee on the Ossification of the Spinal Ligaments of the Japanese Ministry of Health, Labour and Welfare and the Hospital Function Improvement Research of the Japan Organization of Occupational Health and Safety.

\section{References}

1. Chen Y, Guo Y, Chen D, Wang X, Lu X, Yuan W: Long-term outcome of laminectomy and instrumented fusion for cervi- 
cal ossification of the posterior longitudinal ligament. Int Orthop 33:1075-1080, 2009

2. Chiba K, Yamamoto I, Hirabayashi H, Iwasaki M, Goto H, Yonenobu K, et al: Multicenter study investigating the postoperative progression of ossification of the posterior longitudinal ligament in the cervical spine: a new computer-assisted measurement. J Neurosurg Spine 3:17-23, 2005

3. Fujimori T, Iwasaki M, Okuda S, Takenaka S, Kashii M, Kaito T, et al: Long-term results of cervical myelopathy due to ossification of the posterior longitudinal ligament with an occupying ratio of $60 \%$ or more. Spine (Phila Pa 1976) 39:58-67, 2014

4. Goel A, Nadkarni T, Shah A, Rai S, Rangarajan V, Kulkarni A: Is only stabilization the ideal treatment for ossified posterior longitudinal ligament? Report of early results with a preliminary experience in 14 patients. World Neurosurg 84:813-819, 2015

5. Hirabayashi K, Miyakawa J, Satomi K, Maruyama T, Wakano K: Operative results and postoperative progression of ossification among patients with ossification of cervical posterior longitudinal ligament. Spine (Phila Pa 1976) 6:354-364, 1981

6. Iwasaki M, Kawaguchi Y, Kimura T, Yonenobu K: Longterm results of expansive laminoplasty for ossification of the posterior longitudinal ligament of the cervical spine: more than 10 years follow up. J Neurosurg 96 (2 Suppl):180-189, 2002

7. Iwasaki M, Okuda S, Miyauchi A, Sakaura H, Mukai Y, Yonenobu K, et al: Surgical strategy for cervical myelopathy due to ossification of the posterior longitudinal ligament: Part 1: clinical results and limitations of laminoplasty. Spine (Phila Pa 1976) 32:647-653, 2007

8. Kato Y, Iwasaki M, Fuji T, Yonenobu K, Ochi T: Long-term follow-up results of laminectomy for cervical myelopathy caused by ossification of the posterior longitudinal ligament. J Neurosurg 89:217-223, 1998

9. Kawaguchi Y, Kanamori M, Ishihara H, Nakamura H, Sugimori K, Tsuji H, et al: Progression of ossification of the posterior longitudinal ligament following en bloc cervical laminoplasty. J Bone Joint Surg Am 83:1798-1802, 2001

10. Kimura A, Seichi A, Hoshino Y, Yamazaki M, Mochizuki M, Aiba A, et al: Perioperative complications of anterior cervical decompression with fusion in patients with ossification of the posterior longitudinal ligament: a retrospective, multi-institutional study. J Orthop Sci 17:667-672, 2012

11. Koda M, Mochizuki M, Konishi H, Aiba A, Kadota R, Inada $\mathrm{T}$, et al: Comparison of clinical outcomes between laminoplasty, posterior decompression with instrumented fusion, and anterior decompression with fusion for K-line (-) cervical ossification of the posterior longitudinal ligament. Eur Spine J 25:2294-2301, 2016

12. Liu X, Chen Y, Yang H, Li T, Xu B, Chen D: Expansive open-door laminoplasty versus laminectomy and instrumented fusion for cases with cervical ossification of the posterior longitudinal ligament and straight lordosis. Eur Spine J 26:1173-1180, 2017

13. Matsunaga S, Kukita M, Hayashi K, Shinkura R, Koriyama C, Sakou T, et al: Pathogenesis of myelopathy in patients with ossification of the posterior longitudinal ligament. J Neurosurg 96 (2 Suppl):168-172, 2002

14. Nakashima H, Imagama S, Yukawa $Y$, Kanemura T, Kamiya M, Yanase M, et al: Multivariate analysis of C-5 palsy incidence after cervical posterior fusion with instrumentation. $\mathbf{J}$ Neurosurg Spine 17:103-110, 2012
15. Onari K, Akiyama N, Kondo S, Toguchi A, Mihara H, Tsuchiya T: Long-term follow-up results of anterior interbody fusion applied for cervical myelopathy due to ossification of the posterior longitudinal ligament. Spine (Phila Pa 1976) 26:488-493, 2001

16. Saito J, Maki S, Kamiya K, Furuya T, Inada T, Ota M, et al: Outcome of posterior decompression with instrumented fusion surgery for K-line (-) cervical ossification of the longitudinal ligament. J Clin Neurosci 32:57-60, 2016

17. Sakai K, Okawa A, Takahashi M, Arai Y, Kawabata S, Enomoto M, et al: Five-year follow-up evaluation of surgical treatment for cervical myelopathy caused by ossification of the posterior longitudinal ligament: a prospective comparative study of anterior decompression and fusion with floating method versus laminoplasty. Spine (Phila Pa 1976) 37:367376, 2012

18. Smith ZA, Buchanan CC, Raphael D, Khoo LT: Ossification of the posterior longitudinal ligament: pathogenesis, management, and current surgical approaches. A review. Neurosurg Focus 30(3):E10, 2011

19. Takemitsu M, Cheung KM, Wong YW, Cheung WY, Luk KD: C5 nerve root palsy after cervical laminoplasty and posterior fusion with instrumentation. J Spinal Disord Tech 21:267-272, 2008

20. Takenaka S, Nagamoto Y, Aono H, Kaito T, Hosono N: Differences in the time of onset of postoperative upper limb palsy among surgical procedures: a meta-analysis. Spine J 16:1486-1499, 2016

21. Tani T, Ushida T, Ishida K, Iai H, Noguchi T, Yamamoto H: Relative safety of anterior microsurgical decompression versus laminoplasty for cervical myelopathy with a massive ossified posterior longitudinal ligament. Spine (Phila Pa 1976) 27:2491-2498, 2002

22. Yang L, Gu Y, Shi J, Gao R, Liu Y, Li J, et al: Modified plateonly open-door laminoplasty versus laminectomy and fusion for the treatment of cervical stenotic myelopathy. Orthopedics 36:e79-e87, 2013

23. Yoshii T, Sakai K, Hirai T, Yamada T, Inose H, Kato T, et al: Anterior decompression with fusion versus posterior decompression with fusion for massive cervical ossification of the posterior longitudinal ligament with a $\geq 50 \%$ canal occupying ratio: a multicenter retrospective study. Spine J 16:13511357,2016

\section{Disclosures}

The authors report no conflict of interest concerning the materials or methods used in this study or the findings specified in this paper.

\section{Author Contributions}

Conception and design: Nagamoto, Iwasaki. Acquisition of data: Nagamoto. Analysis and interpretation of data: Nagamoto, Sugiura. Drafting the article: Nagamoto. Critically revising the article: Iwasaki, Okuda, Matsumoto, Sugiura, Takahashi, Furuya. Reviewed submitted version of manuscript: Iwasaki, Okuda, Matsumoto, Takahashi, Furuya. Approved the final version of the manuscript on behalf of all authors: Nagamoto. Statistical analysis: Nagamoto. Study supervision: Iwasaki.

\section{Correspondence}

Yukitaka Nagamoto: Osaka Rosai Hospital, Sakai, Japan. 7gam0to@gmail.com. 